\title{
Functional Determinants on Mandelstam Diagrams ${ }^{\star}$
}

\author{
Eric D'Hoker ${ }^{1}$ and D. H. Phong ${ }^{2}$ \\ 1 Department of Physics, University of California, Los Angeles, CA 90024, USA \\ ${ }^{2}$ Department of Mathematics, Columbia University, New York, NY 10027, USA
}

\begin{abstract}
We investigate the special properties of Mandelstam metrics in regard to changing weights in path integrals and relations between determinants of different spins. Regularizations of determinants are discussed along the lines of Sonoda. Weyl anomalies developing at zeroes of metrics in reparametrization invariant regularizations are evaluated in terms of Arakelov metrics. Holomorphic forms are constructed, and determinant identities for Arakelov and Mandelstam metrics rigorously established for any weight and generic even and odd spin structures.
\end{abstract}

\section{Introduction}

Determinants of Laplacians are playing an increasingly important role in diverse areas of physics and geometry. The foundations of their theory have however been developed mostly for compact manifolds and regular metrics. In this paper we wish to examine properties of determinants on certain surfaces with degenerate metrics and punctures which arise as Feynman diagrams in string theory.

It is a fundamental principle of string theory that scattering amplitudes depend only on the conformal class of the Feynman diagrams and not on particular choices of metrics within the class. Nevertheless it is sometimes useful to select a privileged representative to carry out explicit computations. For surfaces, two metrics arise which in some sense are opposites of one another: metrics with constant curvature, and metrics with all curvature concentrated at isolated points. The former are familiar from hyperbolic geometry, and their determinants now well understood in the compact case, as special values of the Selberg zeta function [1]. Examples of the latter are $\left|v_{+}\right|^{4}$ or $\left|\omega_{z}\right|^{2}$, where $v_{+}$and $\omega_{z}$ are respectively a meromorphic spinor [2] and a meromorphic form [3,4]. Their poles can be viewed as punctures on the surface indicating external string states, and their zeroes as interaction

\footnotetext{
* Work supported by the National Science Foundation under grants PHY-80-19754 PHY-86-13201 and DMS-87-04209
} 
points in a Feynman diagram representation. Such surfaces are known as Mandelstam diagrams, and we will refer to their metrics as Mandelstam metrics. For Mandelstam diagrams with at least two punctures, physical parameters given by interaction times, twist angles, internal and external momenta in their natural ranges provide a single copy of the corresponding moduli space. This is basic to a light-cone gauge formulation of string perturbation theory $[3,5]$.

Relations between determinants of different spins for Mandelstam diagrams are central to the issue of unitary of the Polyakov string. At a formal level, a relation between scalar and spin 2 ghost determinants, as well as the equivalence between light-cone gauge and Polyakov approaches for the bosonic string was derived in [6]. A careful treatment of this case together with modifications dictated by regularization procedures was provided later by Sonoda [7]. Formal relations between Dirac and spin 3/2 superghost determinants have been obtained in $[8]$.

In this paper we investigate this type of relations for any spin. We provide a heuristic argument for them in the spirit of Witten's arguments for multiplicative Ward identities [9]. In the present context however, the conformal anomaly is crucial since we deal with path integrals over fields of different spins. Thus the identities hold only for Mandelstam metrics, as it should be expected. At the rigorous level, we follow Sonoda in defining the determinant ratio

$$
\frac{\operatorname{det}^{\prime} \Delta_{n}^{-}}{\operatorname{det}\left\langle\phi_{a}^{n} \mid \phi_{b}^{n}\right\rangle \operatorname{det}\left\langle\phi_{a}^{1-n} \mid \phi_{b}^{1-n}\right\rangle}\left(\left|\omega_{z}\right|^{2}\right)
$$

for Mandelstam metrics by scalings to a regular metric. We show that the reparametrization invariant regularization of the Liouville action develops a Weyl anomaly at each zero of the Mandelstam metric. Elimination of the Weyl anomaly leads to equivalence with Sonoda's coordinate-dependent regularization, and the proper definition for (1.1) is thus completely unambiguous. Bosonization $[10,11,12]$ and Sonoda's methods can then be applied to establish the conjectured identities, for any spin and both odd and even spin structures. For surfaces with punctures, coordinate-dependent regularization is the only reasonable procedure, and the same analysis can be applied to produce similar identities.

Finally we note that for spin $1 / 2$ the determinants of zero modes in (1.1) is finite, so we get a genuine notion of determinants for Laplacians on spinors with respect to Mandelstam metrics. It would be valuable to know whether this notion admits spectral theoretic interpretations or other characterizations besides the identities discussed here. For hyperbolic surfaces with cusps, an interesting candidate for a determinant based on scattering data has been suggested in [13].

\section{Formal Considerations}

We shall be mainly concerned with determinants of Laplacians acting on fields $b, c$ of ranks $n$ and $1-n$ on a Riemann surface $M$. More precisely the CauchyRiemann operator $\partial_{\bar{z}}$ sends $b(d z)^{n}$ and $c(d z)^{1-n}$ to $\partial_{\bar{z}} b d \bar{z} d z^{n}$ and $\partial_{\bar{z}} c d \bar{z} d z^{1-n}$. If we choose a metric $d s^{2}=2 g_{z z} d \bar{z} d z$ to represent the complex structure of $M$, then the covariant derivatives $\nabla_{z}^{n}$ and $\nabla_{n}^{z}$ can be written as 


$$
\nabla_{n}^{z}\left(b(d z)^{n}\right)=g^{z \bar{z}} \partial_{z} b d z^{n-1}, \quad \nabla_{z}^{n}\left(b(d z)^{n}\right)=g_{z \bar{z}}^{n} \partial_{z}\left(\left(g^{z \bar{z}}\right)^{n} b\right) d z^{n+1} .
$$

With respect to the pairing

$$
\|b\|^{2}=\int d^{2} z\left(g^{z \bar{z}}\right)^{n-1} \bar{b} b, \quad\|c\|^{2}=\int d^{2} z\left(g^{z \bar{z}}\right)^{-n} \bar{c} c
$$

it is readily seen that $\left(\nabla_{n}^{z}\right)^{\dagger}=-\nabla_{z}^{n-1},\left(\nabla_{z}^{n}\right)^{\dagger}=-\nabla_{n+1}^{z}$, and we can form the Laplacians

$$
\Delta_{n}^{+}=-2 \nabla_{n+1}^{z} \nabla_{z}^{n}, \quad \Delta_{n}^{-}=-2 \nabla_{z}^{n-1} \nabla_{n}^{z} .
$$

It is evident that the spectra of $\Delta_{n}^{-}, \Delta_{-n}^{+}$, and $\Delta_{1-n}^{-}$are identical, so henceforth we shall restrict to determinants of $\Delta_{n}^{-}$for $1 / 2 \leqq n$. For $n$ half-integer, proper definition of rank $n$ tensors requires selection of a spin structure on $M$, and we always assume this has been done. The genus of $M$ will be denoted by $h$.

The relations between determinants of different weights we are looking for are suggested by a change of variables in the chirally symmetric path integrals over anti-commuting fields $b$ and $c$

$$
Z_{n}\left(z_{1}, \ldots, w_{M}\right)=\int D(b \bar{b} c \bar{c}) \exp \left(-I_{n}(b, c)\right)\left|\prod_{i=1}^{M+r_{n}} b\left(z_{i}\right) \prod_{j=1}^{M} c\left(w_{j}\right)\right|^{2}
$$

Here the action is given by

$$
I_{n}(b, c)=\frac{1}{2 \pi} \int d^{2} z\left(b \partial_{\bar{z}} c+\text { c.c }\right) .
$$

and $Y_{n}$ is the index of the Laplacian $\Delta_{n}^{-}$which gives the violation of fermion number. Recall that the Riemann-Roch theorem asserts that

$$
Y_{n}=(\# b \text { zeromodes })-(\# c \text { zeromodes })=-\frac{1}{2}(2 n-1) \chi(M),
$$

where the dimensions are taken over complex numbers. To pass from a rank $n$ tensor $b$ to a rank $n+1$ tensor $b^{\prime}$ we need a holomorphic one-form $\omega_{z}$ so as to set

$$
b^{\prime}=\omega_{z} b, \quad c^{\prime}=\omega_{z}^{-1} c .
$$

For this transformation to be an isometry with respect to the pairing (2.2), the metric $d s^{2}=2 g_{z \bar{z}} d z d \bar{z}$ cannot be any metric but must be chosen to be the singular metric

$$
2\left|\omega_{z}\right|^{2}|d z|^{2} \text {. }
$$

We shall refer to metrics of the form (2.8) as Mandelstam metrics. A holomorphic form will have $2 h-2$ zeroes, and we shall assume for simplicity that the zeroes of $\omega_{z}$ are simple, and denote them by $R_{a}$. To preserve locality of the path integrals, we must require $c^{\prime}$ to be continuous, $b^{\prime}$ to vanish at $R_{a}$. Vanishing of the fermionic field $b^{\prime}$ at $R_{a}$ can be insured by an insertion of $b^{\prime}\left(R_{a}\right)$, and we are thus led to

$$
\int D\left(b^{\prime} \bar{b}^{\prime} c^{\prime} \bar{c}^{\prime}\right) \exp \left(-I_{n+1}\left(b^{\prime}, c^{\prime}\right)\right)\left|\prod_{1}^{M+r_{n}} b^{\prime}\left(z_{i}\right) \prod_{1}^{M} c^{\prime}\left(\omega_{j}\right) \prod_{1}^{2 h-2} b^{\prime}\left(R_{a}\right)\right|^{2}\left|\frac{\prod_{1}^{M} \omega_{w_{j}}}{\prod_{1}^{M+r_{n}} \omega_{z_{l}}}\right|^{2} .
$$


Note that the fermion number violation is now $r_{n}+(2 h-2)=r_{n+1}$, as it should be. However the expression (2.9) cannot agree with the original expression (2.4) since their tensor types at $R_{a}$ are different. To remedy the situation, we introduce powers of the non-vanishing rank two tensor at $R_{a}$,

$$
\partial \omega\left(R_{a}\right)
$$

The naive powers to go with (2.9) would seem to be $\left|\partial \omega\left(R_{a}\right)\right|^{-(n+1)}$, and they would be correct if the determinants of $\Delta_{n}^{-}$were scalars as in the case of regular metrics. Here the requirement that (2.7) be an isometry has forced us to take singular Mandelstam metrics (2.8). We shall see in Sect. 4 that any regularization consistent with Weyl scalings will force the determinant ratio

$$
W_{n}\left(\left|\omega_{z}\right|^{2}\right)=\frac{\operatorname{det}^{\prime} \Delta_{n}^{-}}{\operatorname{det}\left\langle\phi_{a}^{n} \mid \phi_{b}^{n}\right\rangle \operatorname{det}\left\langle\phi_{a}^{1-n} \mid \phi_{b}^{1-n}\right\rangle}
$$

to transform as a tensor of $\operatorname{rank}\left(c_{n} / 12, c_{n} / 12\right)$ at each zero $R_{a}$ of the metric. Here

$$
c_{n}=6 n^{2}-6 n+1
$$

is the familiar coefficient of the conformal anomaly, and $\phi_{a}^{n}$ are a fixed basis of zero modes for $\nabla_{n}^{z}$. It follows that the proper correction factor is $\left|\partial \omega\left(R_{a}\right)\right|^{-(n+1)-\left(c_{n+1}-c_{n}\right) / 12}=\left|\partial \omega\left(R_{a}\right)\right|^{-2 n-1}$. Thus the change of weight formula in path integrals should be

$$
\begin{aligned}
\int D(b \bar{b} c \bar{c}) \exp \left(-I_{n}\right)\left|\prod_{1}^{M+r_{n}} b\left(z_{i}\right) \prod_{1}^{M} c\left(w_{j}\right)\right|^{2}= & \int D(b \bar{b} c \bar{c}) \exp \left(-I_{n+1}\right) \\
& \cdot\left|\prod_{1}^{M+r_{n}} b\left(z_{i}\right) \prod_{1}^{2 h-2} b\left(R_{a}\right) \prod_{1}^{M} c\left(w_{j}\right)\right|^{2} \\
& \cdot\left|\frac{\prod_{1}^{M} \omega\left(w_{j}\right)}{\mid \frac{T_{n}}{\prod_{1}} \omega\left(z_{i}\right)}\right|^{2}\left|\prod_{1}^{2 h-2} \partial \omega\left(R_{a}\right)\right|^{-2 n-1} .
\end{aligned}
$$

To derive relations between determinants we consider (2.12) with the minimal number of insertions to absorb all zero modes. For simplicity we discuss now only the case of weight $n>1$, so that there are no $c$-zero mode, the number of $b$-zero modes is $r_{n}$, and we may set $M=0$. The result is

$$
\begin{aligned}
\frac{\operatorname{det}^{\prime} \Delta_{n}^{-}}{\operatorname{det}\left\langle\phi_{a}^{n} \mid \phi_{b}^{n}\right\rangle}\left|\operatorname{det} \phi_{a}^{n}\left(z_{b}\right)\right|^{2}= & \frac{\operatorname{det}^{\prime} \Delta_{n+1}^{-}}{\operatorname{det}\left\langle\phi_{a}^{n+1} \mid \phi_{b}^{n+1}\right\rangle}\left|\operatorname{det} \phi_{a}^{n+1}\left(z_{b}, R_{a}\right)\right|^{2} \\
& \cdot \prod_{1}^{2 n-2}\left|\partial \omega\left(R_{a}\right)\right|^{-2 n-1} \prod_{1}^{r_{n}}\left|\omega\left(z_{i}\right)\right|^{-2} .
\end{aligned}
$$

It is worth observing that with the regularization we shall adopt for the determinant ratio $\left.\left.W_{n}\left(\mid \omega_{z}\right)\right|^{2}\right)$ will scale as

$$
W_{n}\left(e^{2 \mu}\left|\omega_{z}\right|^{2}\right)=W_{n}\left(\left|\omega_{z}\right|^{2}\right) \exp \left(\frac{2}{3}(h-1) c_{n} \mu\right)
$$


for constant $\mu$ and fixed choice of zero modes, so that (2.13) is indeed independent of any normalization for the holomorphic form $\omega_{z}$. It would otherwise carry little information.

The relation (2.13) can be simplified considerably if we choose the bases $\phi_{a}^{n}$ and $\phi_{a}^{n+1}$ appropriately. First we need a propagator, i.e., a rank $n$ form meromorphic form in $z$ with a simple pole at $w$. Evidently two such propagators will differ by a holomorphic $n$ form, and thus such propagators are parametrized by $r_{n}$ parameters. If we choose these parameters to be $r_{n}$ generic points on the Riemann surface $M$, we can construct the corresponding propagator explicitly as

$$
\begin{aligned}
S^{n}\left(z, w ; z_{1}, \ldots, z_{r_{n}}\right) & =\frac{\int D(b c) \exp \left(-I_{n}(b, c)\right) b(z) c(w) \prod_{1}^{r_{n}} b\left(z_{i}\right)}{\int D(b c) \exp \left(-I_{n}(b, c)\right) \prod_{1}^{r_{n}} b\left(z_{i}\right)} \\
& =\frac{\left\langle b(z) c(w) \prod_{1}^{r_{n}} b\left(z_{i}\right)\right\rangle}{\left\langle\prod_{1}^{r_{n}} b\left(z_{i}\right)\right\rangle} .
\end{aligned}
$$

Then $S^{n}\left(z, \omega ; z_{1}, \ldots, z_{Y_{n}}\right)$ is a rank $n$ tensor in $z$ with a unique pole at $w$ and zeroes at $z_{i}$. Given a basis $\phi_{a}^{n}$ of holomorphic rank $n$ tensors, we can construct a basis for holomorphic rank $n+1$ tensors by letting

$$
\begin{aligned}
\phi_{a}^{n+1}(z) & =\omega_{z} \phi_{a}^{n}(z), \quad a=1, \ldots, \Upsilon_{n}, \\
\phi_{Y_{n}+b}^{n+1}(z) & =\omega_{z} S^{n}\left(z, R_{b} ; z_{1}, \ldots, z_{Y_{n}}\left(\partial \omega\left(R_{b}\right)\right)^{(n-1) / 2} .\right.
\end{aligned}
$$

In (2.16) the factor $\left(\partial \omega\left(R_{b}\right)\right)^{(n-1) / 2}$ is necessary to insure that $\phi_{Y_{n}+b}^{n+1}$ be a rank $n+1$ tensor. The reason is that $S^{n}\left(z, w ; z_{1}, \ldots, z_{Y_{n}}\right)$ is a rank $1-n$ tensor in $w$, so that $\lim _{z \rightarrow R_{b}} \omega_{z} S^{n}\left(z, R_{b} ; z_{1}, \ldots, z_{Y_{n}}\right)$ is a rank 2 tensor at $R_{b}$ for any weight $n$. It is now immediately seen that the matrix $\phi_{c}^{n+1}\left(z_{i}, R_{b}\right)$ is block diagonal, and

$$
\frac{\operatorname{det} \phi_{c}^{n+1}\left(z_{i}, R_{b}\right)}{\operatorname{det} \phi_{a}^{n}\left(z_{i}\right)}=\prod_{1}^{2 h-2} \partial \omega\left(R_{b}\right)^{(n+1) / 2} \prod_{1}^{r_{n}} \omega\left(z_{i}\right) .
$$

Thus we obtain the remarkable relation

$$
\frac{\operatorname{det}^{\prime} \Delta_{n}^{-}}{\operatorname{det}\left\langle\phi_{a}^{n} \mid \phi_{b}^{n}\right\rangle}=\frac{\operatorname{det}^{\prime} \Delta_{n+1}^{-}}{\operatorname{det}\left\langle\phi_{a}^{n+1} \mid \phi_{b}^{n+1}\right\rangle}\left|\prod_{1}^{2 h-2} \partial \omega\left(R_{a}\right)\right|^{-n} .
$$

With the regularizations described in Sect. 4, we shall establish later by explicit computations that (2.18) does hold for Mandelstam metrics $d s^{2}=2\left|\omega_{z}\right|^{2}|d z|^{2}$, together with its analogues for weights $n=1$ and $n=1 / 2$, and for both types of spin structures. The case $n=1$ is the one treated in Sonoda [7]. The presence of the factor involving $\partial \omega\left(R_{a}\right)$ in (2.18) is required by the fact that $W_{n}\left(\left|\omega_{z}\right|^{2}\right)$ is a tensor of rank $\left(c_{n} / 12, c_{n} / 12\right)$ at each $R_{a}$. Perhaps more important, the above discussion shows that this factor also leads to invariance of (2.18) under constant scalings of $\omega_{z}$. For rank $n=1$ the relation (2.16) between bases of holomorphic differentials 
and quadratic differentials simplifies, and this key scale invariance in $\omega_{z}$ of (2.18) can be checked directly, assuming the reasonable behavior of $W_{n}\left(\left|\omega_{z}\right|^{2}\right)$ given by (2.14).

\section{Arakelov Green's Functions and Arakelov Metrics}

To regularize determinants with respect to the metric $\left|\omega_{z}\right|^{2}$, we shall scale $\left|\omega_{z}\right|^{2}$ to a regular metric. Conformal factors between metrics of given curvatures and their Liouville actions are most conveniently expressed in terms of Arakelov Green's functions, so we begin with a brief study of their basic properties and scaling behavior.

Let $d s^{2}=2 g_{z \bar{z}} d z d \bar{z}$ be a fixed metric on the surface $M$. The usual scalar Green's function $G(z, w)$ is symmetric and characterized by

$$
\begin{gathered}
-\partial_{z} \partial_{\bar{z}} G(z, w)=2 \pi \delta(z-w)-\frac{2 \pi g_{z \bar{z}}}{\int d^{2} z \sqrt{g}}, \\
\int d^{2} z g_{z \bar{z}} G(z, w)=0 .
\end{gathered}
$$

The Arakelov Green's function $G^{A}(z, w)$ on the other hand will be defined by

$$
\begin{gathered}
-\partial_{z} \partial_{\bar{z}} G^{A}(z, w)=2 \pi \delta(z-w)+\frac{g_{z \bar{z}} R}{2(h-1)}, \\
\int d^{2} z g_{z \bar{z}} R G^{A}(z, w)=0
\end{gathered}
$$

where $R=-g^{z \bar{z}} \partial_{z} \partial_{\bar{z}} \ln g_{z \bar{z}}$ is the curvature scalar. Equation (3.4) is just a normalization condition. We note that $G^{A}(z, w)$ is invariant if we scale $g_{z \bar{z}}$ by a constant. Solving for $G^{A}(z, w)$ in terms of the usual Green's function $G(z, w)$ gives

$$
\begin{aligned}
G^{A}(z, w)= & G(z, w)+\frac{1}{4 \pi(h-1)} \int d^{2} v G(z, v) g_{v \bar{v}} R+\frac{1}{4 \pi(h-1)} \int d^{2} v G(v, w) g_{v \bar{v}} R \\
& +\frac{1}{16 \pi^{2}(h-1)^{2}} \int d^{2} v d^{2} u g_{v \bar{v}} R G(v, u) g_{u \bar{u}} R
\end{aligned}
$$

which shows that the Arakelov Green's function is symmetric,

$$
G^{A}(z, w)=G^{A}(w, z) .
$$

Let now $d \hat{s}^{2}=2 \hat{g}_{z \bar{z}} d z d \bar{z}$ be another metric conformally equivalent to $d s^{2}$, with

$$
g_{z \bar{z}}=\exp (2 \lambda) \hat{g}_{z \bar{z}} \text {. }
$$

If $G^{A}(z, w)$ denotes the corresponding Arakelov Green's function, it is readily seen that the same equation (3.5) will hold, with $G(z, w)$ replaced by $\hat{G}^{A}(z, w)$ on the right-hand side. Since the curvatures of the two metrics are related by

$$
g_{z \bar{z}} R=\hat{g}_{z \bar{z}} \hat{R}-2 \partial_{z} \partial_{\bar{z}} \lambda .
$$

we can evaluate the right-hand side explicitly and obtain

$$
G^{A}(z, w)=\hat{G}^{A}(z, w)+\frac{1}{(h-1)}(\lambda(z)+\lambda(w))+\frac{6}{(h-1)^{2}} S(\hat{g}, \lambda) .
$$


Here $S(\hat{g}, \lambda)$ is the Liouville action

$$
S(\hat{g}, \lambda)=\frac{1}{12 \pi} \int d^{2} z\left(\lambda \partial_{z} \partial_{\bar{z}} \lambda+\hat{g}_{z \bar{z}} \hat{R} \lambda\right)
$$

At coincident points, Green's functions will diverge as $-\ln d^{2}(z, w)$, where $d(z, w)$ is the reparametrization invariant distance with respect to the metric $d s^{2}$. Thus we may regularize the Green's function $G^{A}(z, w)$ by

$$
G^{A}(z, z)=\lim _{w \rightarrow z}\left(G^{A}(z, w)+\ln d^{2}(z, w)\right) .
$$

Note that $G^{A}(z, z)$ is a reparametrization invariant scalar. For coincident points the scaling law (3.8) becomes

$$
G^{A}(z, z)=\hat{G}^{A}(z, z)+\frac{2 h}{(h-1)} \lambda(z)+\frac{6}{(h-1)^{2}} S(\hat{g}, \lambda),
$$

since $d^{2}(z, w)=\hat{d}^{2}(z, w) e^{2 \lambda(z)}$ up to higher order terms for $z$ near $w$.

We can now eliminate the explicit dependence on the genus by forming combinations of Arakelov Green's functions. The simplest such expression is

$$
\left(4 g_{z \bar{z}} g_{w \bar{w}}\right)^{-1 / 2} \exp \left(-G^{A}(z, w)+\frac{1}{2} G^{A}(z, z)+\frac{1}{2} G^{A}(w, w)\right),
$$

which is immediately seen to agree with the similar expression in terms of the Green's function $G(z, w)$ and its reparametrization invariant regularization $G(z, z)=\lim _{w \rightarrow z}\left(G(z, w)+\ln d^{2}(z, w)\right)$. This is known to be the same as the $(-1 / 2,-1 / 2) \times(-1 / 2,-1 / 2)$ form on $M \times M$

$$
F(z, w)=\exp \left(-2 \pi \sum_{I J} \operatorname{Im} \int_{w}^{z} \omega_{I}(\operatorname{Im} \Omega)_{I J}^{-1} \operatorname{Im} \int_{w}^{z} \omega_{J}\right)|E(z, w)|^{2},
$$

where $E(z, w)$ is the prime form, $\omega_{I}$ a basis of abelian differentials, and $\Omega_{I J}$ the corresponding period matrix. A more unusual combination is obtained by selecting any $(2 h-2)$ points $R_{a}$ on the surface $M$, and considering

$$
\sum_{a, b=1}^{2 h-2} G^{A}\left(R_{a}, R_{b}\right)
$$

Unless stated explicitly otherwise, coincident points are included at which the Arakelov Green's functions are taken to be their regularized values. The expression (3.13) now transforms as

$$
\sum_{a, b=1}^{2 h-2} G^{A}\left(R_{a}, R_{b}\right)=\sum_{a, b=1}^{2 h-2} \hat{G}^{A}\left(R_{a}, R_{b}\right)+6 \sum_{a, b=1}^{2 h-2} \lambda\left(R_{a}\right)+24 S(\hat{g}, \lambda) .
$$

This equation will be needed when evaluating the Weyl anomaly developing at each zero $R_{a}$ of a Mandelstam metric $\left|\omega_{z}\right|^{2} \sim\left|\partial \omega\left(R_{a}\right)\right|^{2}\left|z-R_{a}\right|^{2}$.

So far we have discussed Arakelov Green's functions with respect to any metric $d s^{2}=2 g_{z \bar{z}} d z d \bar{z}$. Some calculations simplify if we make use of some particular metric on the surface $M$. Recall that $M$ can be imbedded in its Jacobian $C^{h} /\left(Z^{h}+\Omega Z^{h}\right)$ by 
the Abel map

$$
z \rightarrow \int_{P}^{z} \omega_{I},
$$

where $P$ is a fixed base point. The induced metric on $M$ from the flat metric on the Jacobian is

$$
j_{z \bar{z}}=\sum_{I J} \omega_{I}(z)(\operatorname{Im} \Omega)_{I J}^{-1} \bar{\omega}_{J}(z) .
$$

The Arakelov metric on $M$ is a metric $g_{z \bar{z}}^{A}$ whose curvature form $g_{z \bar{z}}^{A} R^{A}$ is equal to $2 \pi(1-h) j_{z \bar{z}} / h$. This condition determines $g_{z \bar{z}}^{A}$ only up to a multiplicative constant which we shall choose later. It is then evident that the Arakelov Green's function $G^{A}(z, w)$ with respect to the Arakelov metric is the same as the usual Green's function with respect to the metric $j_{z \bar{z}}$ induced from the Jacobian.

This process of passing from a metric to another metric whose curvature is proportional to the volume form of the former can in principle be repeated indefinitely. With suitable normalizations of the volume, it will converge to constant curvature metrics.

Returning to the Arakelov metric proper, we note that its Arakelov Green's function is actually rather simple. Since the form $F(z, w)$ of $(3.12)$ satisfies the equation

$$
\partial_{z} \partial_{\bar{z}} \ln F(z, w)=2 \pi \delta(z, w)-\pi j_{z \bar{z}}
$$

the Arakelov Green's function for the Arakelov metric must be equal to

$$
G^{A}(z, w)=-\ln F(z, w)-\frac{1}{2} \ln g_{z \bar{z}}^{A}-\frac{1}{2} \ln g_{w \bar{w}}^{A}
$$

up to an additive constant independent of both $z$ and $w$. This implies that up to an additive constant we have

$$
-\ln g_{z \bar{z}}^{A}=\lim _{w \rightarrow z}\left(G^{A}(z, w)+\ln |z-w|^{2}\right) .
$$

The initial constant ambiguity in the definition of the Arakelov metric can now be eliminated by requiring that (3.18), and hence (3.17), hold exactly as they are written there. The relations (3.17), (3.18) are sometimes referred to as residue formulas. The usefulness of Arakelov metrics and their Arakelov Green's functions in bosonization can now be traced to the fact that $F(z, w)$ is essentially the correlation function of two normal-ordered vertex operators

$$
\left(2 g_{z \bar{z}}\right)^{k^{2} / 2}\left(2 g_{w \bar{w}}\right)^{\left(k^{\prime}\right)^{2} / 2}\left\langle: e^{i k x(z)}:: e^{i k^{\prime} x(w)}:\right\rangle=\delta\left(k+k^{\prime}\right) F(z, w)^{-k^{2}}
$$

in a bose theory of scalar fields $x$ with lagrangian $\partial_{z} x \partial_{\bar{z}} x / 4 \pi$. In Arakelov's original work [14] norms on line bundles are defined by setting $\left\|1_{w}\right\|=\exp \left(-G^{A}(z, w)\right)$ for the canonical section $1_{w}$ of the point bundle $O(w)$. The above relations then express the fact that for the canonical bundle the new metric defined this way will give back the Arakelov metric.

The right-hand side of (3.18) is a regularization procedure for $G^{A}(z, w)$ at coincident points which is coordinate dependent, but does not require a choice of metric within the conformal class. We shall sometimes denote it by : $G^{A}(z, z)$ : to distinguish it from the $G^{A}(z, z)$ of (3.10). Note that exp: $G^{A}(z, z)$ : is a $(-1,-1)$ 
tensor. For the Arakelov metric, the reparametrization invariant regularization $G^{A}(z, z)$ vanishes

$$
G^{A}(z, z)=0 .
$$

This follows from (3.18) and the fact that the distance $d^{A}(z, w)$ equals $|z-w|\left(2 g_{z \bar{z}}^{A}\right)^{1 / 2}$ up to higher order terms.

This completes our survey of Arakelov Green's functions.

\section{Liouville Actions and Regularization of Determinants}

We turn now to the problem of defining a determinant for the surface $M$ with a Mandelstam metric $\left|\omega_{z}\right|^{2}$, where $\omega_{z} d z$ is a holomorphic abelian differential. Following Sonoda [7] we introduce a regular (i.e. with neither zeros nor poles) metric $g_{z \bar{i}}$ conformally equivalent to $\left|\omega_{z}\right|^{2}$,

$$
\left|\omega_{z}\right|^{2}=\exp (2 \sigma(z)) g_{z \bar{z}}
$$

Taking the curvatures for both sides gives an equation for $\sigma(z)$, which can be solved using the Arakelov Green's function $G^{A}(z, w)$ for the metric $g_{z \bar{z}}$

$$
\sigma(z)=-\frac{1}{2} \sum_{a=1}^{2 h-2} G^{A}\left(z, R_{a}\right)-\frac{1}{2} c .
$$

Here $R_{a}, a=1, \ldots, 2 h-2$ are the zeroes of $\omega_{z}$ which are assumed to be simple, and $c$ is a constant measuring the relative normalizations of $\left|\omega_{z}\right|^{2}$ and $g_{z \bar{z}}$ :

$$
c=\frac{1}{2(h-1)}\left(-\sum_{a, b=1}^{2 h-2} G^{A}\left(R_{a}, R_{b}\right)+\ln \left|\prod_{a=1}^{2 h-2} \sqrt{2} g_{R_{a} \overline{\bar{R}}_{a}} / \partial \omega\left(R_{a}\right)\right|^{2}\right) .
$$

Formally Weyl scalings of determinants (more precisely of ratios $W_{n}(g)$ of $(2.11)$ ) would suggest the following definition for $W_{n}\left(\left|\omega_{z}\right|^{2}\right)$ :

$$
W_{n}\left(\left|\omega_{z}\right|^{2}\right)=W_{n}(g) \exp \left(-2 c_{n} S(g, \sigma)\right)
$$

for fixed zero modes, $S(g, \sigma)$ the Liouville action of (3.9), and

$$
c_{n}=6 n^{2}-6 n+1
$$

the coefficient of the conformal anomaly for tensors of rank $n$. However the Liouville action diverges logarithmically for scaling factors $\sigma(z)$ of the type (4.2) which have logarithmic singularities at the points $R_{a}$. There are then two ways of regularizing the Liouville action which we shall examine in turn:

- Cutting out a reparametrization invariant, but metric dependent disk $D_{g}\left(R_{a}\right)=$ $\left\{d\left(z, R_{a}\right)<\varepsilon\right\}$ around each $R_{a}$,

$$
S_{\mathrm{cov}}(g, \sigma)=\lim _{\varepsilon \rightarrow 0} \int_{M(\varepsilon, g)} d^{2} z\left(\partial_{z} \sigma \partial_{\bar{z}} \sigma-\left(\partial_{\bar{z}} \partial_{\bar{z}} \ln g_{z \bar{z}}\right) \sigma\right)-\frac{1}{24}(2 h-2) \ln \varepsilon^{2} .
$$

The surface $M(\varepsilon, g)$ is the surface $M$ with the disks $D_{g}\left(R_{a}\right)$ removed.

The regularized Liouville action (4.5) can actually be computed explicitly in terms of Arakelov Green's functions using the expression (4.2) for the conformal 
factor $\sigma(z)$ :

$$
\begin{aligned}
S_{\mathrm{cov}}(g, \sigma)= & \lim _{\varepsilon \rightarrow 0} \frac{1}{12 \pi} \int_{M(\varepsilon, g)} d^{2} z \sum_{a, b=1}^{2 h-2} \partial_{z} G^{A}\left(z, R_{a}\right) \partial_{\bar{z}} G^{A}\left(z, R_{b}\right) \\
& -\frac{1}{24}(2 h-2) \ln \varepsilon^{2}+\frac{1}{6}(h-1) c .
\end{aligned}
$$

An integration by parts produces

$$
\begin{aligned}
S_{\mathrm{cov}}(g, \sigma)= & \lim _{\varepsilon \rightarrow 0} \frac{1}{12 \pi} \int_{M(\varepsilon, g)} d^{2} z \frac{1}{4} \sum_{a, b=1}^{2 h-2}\left(-\partial_{\bar{z}} \partial_{z} G^{A}\left(z, R_{a}\right)\right) G^{A}\left(z, R_{b}\right) \\
& +\frac{i}{48 \pi} \sum_{a=1}^{2 h-2} \int_{c D_{g}\left(R_{a}\right)} \partial_{z} G^{A}\left(z, R_{a}\right) \sum_{b=1}^{2 h-2} G^{A}\left(z, R_{b}\right) \\
& -\frac{1}{24}(2 h-2) \ln \varepsilon^{2}+\frac{1}{6} c(h-1) .
\end{aligned}
$$

The first term on the right-hand side vanishes due to the normalization condition (3.4) and the fact that $M(\varepsilon, g)$ does not contain any of the sources $R_{a}$. As for the second we may replace $G^{A}\left(z, R_{a}\right)$ effectively by its asymptotic $-\ln \left|z-R_{a}\right|^{2}$. For $b \neq a G^{A}\left(z, R_{b}\right)$ can be replaced by $G^{A}\left(R_{a}, R_{b}\right)$, while for $b=a$ it should be replaced by $-\ln \varepsilon^{2}+G^{A}\left(R_{a}, R_{a}\right)$, with $G^{A}\left(R_{a}, R_{a}\right)$ the reparametrization invariant regularized Arakelov Green's function at coincident points. The result is

$$
S_{\mathrm{cov}}(g, \sigma)=\frac{1}{24} \sum_{a, b=1}^{2 h-2} G^{A}\left(R_{a}, R_{b}\right)+\frac{1}{6}(h-1) c
$$

with $c$ given by (4.3). In particular we can read off the scaling behavior of the Liouville action, under a change from $g$ to $\hat{g}=\exp (-2 \lambda) g$ with $\lambda$ a regular function. Since the normalization constants will scale as

$$
\frac{1}{6} \hat{c}(h-1)-2 S(\hat{g}, \lambda)-\frac{1}{6} \sum_{a=1}^{2 h-2} \lambda\left(R_{a}\right)=\frac{1}{6} c(h-1)
$$

and Arakelov Green's functions scale as in (3.14), we see that the usual additive rule for Liouville actions in the case of regular metrices $g, \hat{g}$ and regular scalings $\lambda, \hat{\sigma}, \sigma=\hat{\sigma}-\lambda$,

$$
S(\hat{g}, \hat{\sigma})=S(\hat{g}, \lambda)+S(g, \sigma)
$$

gets modified to

$$
S_{\mathrm{cov}}(\hat{g}, \hat{\sigma})=S(\hat{g}, \lambda)+S_{\mathrm{cov}}(g, \sigma)-\frac{1}{12} \sum_{a=1}^{2 h-2} \lambda\left(R_{a}\right)
$$

for scalings $\sigma$ of the form (4.2). This particular relation can also be derived directly from (4.5). It can be interpreted as a Weyl anomaly developing at each singular point $R_{a}$ in a metric.

The net outcome is that the naive definition (4.4), (4.5) for the determinant ratio $W_{n}\left(\left|\omega_{z}\right|^{2}\right)$ leads to a scalar which depends on the choice of the regularizing metric $g_{z \bar{z}}$. We can restore independence from $g_{z \bar{z}}$ by defining instead 


$$
\begin{aligned}
W_{n}\left(\left|\omega_{z}\right|^{2}\right)= & W_{n}\left(g_{z \bar{z}}\right) \exp \left(-2 c_{n} S(g, \sigma)\right)\left(\prod_{a=1}^{2 h-2} g_{R_{a} \bar{R}_{a}}\right)^{c_{n} / 12} \\
= & W_{n}\left(g_{z \bar{z}}\right) \exp \left(\frac{c_{n}}{12} \sum_{a, b=1}^{2 h-2} G^{A}\left(R_{a}, R_{b}\right)\right) \\
& \cdot\left(\prod_{a=1}^{2 h-2}\left(\sqrt{2} g_{R_{a} \bar{R}_{a}}\right)^{-1 / 4} \prod_{a=1}^{2 h-2}\left|\partial \omega\left(R_{a}\right)\right|^{1 / 3}\right)^{c_{n}}
\end{aligned}
$$

which is now a tensor of $\operatorname{rank}\left(c_{n} / 12, c_{n} / 12\right)$ at each $R_{a}, a=1, \ldots, 2 h-2$.

- Cutting out Weyl invariant, but coordinate dependent disks $D\left(R_{a}\right)=\left\{\left|z-R_{a}\right|<\varepsilon\right\}$. This is the choice of Sonoda, and letting $M(\varepsilon)=M-\left(D\left(R_{a}\right)\right)$, the Liouville action is regularized by

$$
S(g, \sigma)=\lim _{\varepsilon \rightarrow 0} \frac{1}{12 \pi} \int_{M(\varepsilon)} d^{2} z\left(\partial_{z} \partial_{\bar{z}} \sigma-\left(\partial_{z} \partial_{\bar{z}} \ln g_{z \bar{z}}\right) \sigma\right)-\frac{1}{24}(2 h-2) \ln \varepsilon^{2} .
$$

It is easy to see that the additive rule (4.9) is now unchanged, and $\exp \left(-2 c_{n} S(g, \sigma)\right)$ is a rank $\left(c_{n} / 12, c_{n} / 12\right)$ tensor at each $R_{a}$. This means that $W_{n}\left(\left|\omega_{z}\right|^{2}\right)$ defined by (4.4) and (4.12) is again a rank $\left(c_{n} / 12, c_{n} / 12\right)$ tensor which is independent of the choice of the regular metric $g_{z \bar{z}}$. The same calculation leading to (4.7) now gives

$$
S(g, \sigma)=\frac{1}{24} \sum_{a \neq b} G^{A}\left(R_{a}, R_{b}\right)+\frac{1}{24} \sum_{a=1}^{2 h-2}: G^{A}\left(R_{a}, R_{a}\right):+\frac{1}{6}(h-1) c
$$

from which the additive rule can also be verified using (3.8). We can then show that the two regularizations (4.11) and (4.4), (4.12) lead to identical notions. Since both are independent of $g_{z \bar{z}}$, we may choose $g_{z \bar{z}}$ to be the Arakelov metric $g_{z \bar{z}}^{A}$, upon which $G^{A}\left(R_{a}, R_{a}\right)=0$ and $: G^{A}\left(R_{a}, R_{a}\right):=-\ln g_{z \bar{z}}^{A}$. We then obtain for both regularizations the same answer

$$
W_{n}\left(\left|\omega_{z}\right|^{2}\right)=W_{n}\left(g_{z \bar{z}}^{A}\right) \exp \left(-\frac{c_{n}}{12} \sum_{a, b=1}^{2 h-2} G^{A}\left(R_{a}, R_{b}\right)+\frac{c_{n}}{3}(1-h) c\right)\left(\prod_{a=1}^{2 h-2} g_{R_{a} \bar{R}_{a}}^{A}\right)^{c_{n} / 12}
$$

Thus Weyl scaling rules lead to an unambiguous notion of determinants for Mandelstam metrics.

\section{Evaluations in Terms of Theta Functions}

The goal of this section is to justify the formal rules of Sect. 2 by evaluating explicitly the regularized determinants of Sect. 4, using bosonization formulas.

Bosonization expresses correlation functions of the form (2.4) in terms of the function $F(z, w)$ of (3.12) and (3.19), or equivalently in view of (3.17), (3.18),

$$
\left(g_{z_{i} \bar{z}_{i}}^{A}\right)^{-n}\left(g_{w_{j} \bar{w}_{j}}^{A}\right)^{-1+n} Z_{n}\left(z_{1}, \ldots, w_{M}\right)
$$

in terms of Arakelov Green's functions for the Arakelov metric [10-12], 


$$
\begin{aligned}
\prod & \left(g_{z_{i} \bar{z}_{i}}^{A}\right)^{-n}\left(g_{w_{j} \bar{w}_{j}}^{A}\right)^{-1+n} Z_{n}\left(z_{1}, \ldots, w_{M}\right) \\
= & {\left[\frac{\operatorname{det}^{\prime} \Delta_{0}^{-}}{\int d^{2} z \sqrt{g_{z \bar{z}}^{A}} \operatorname{det} \operatorname{Im} \Omega}\right]^{-1 / 2}|\theta[\delta](0, \Omega)|^{2} } \\
& \cdot \exp \left(-\frac{1}{2} \sum_{i, j} G^{A}\left(z_{i}, z_{j}\right)-\frac{1}{2} \sum_{i, j} G^{A}\left(w_{i}, w_{j}\right)+\sum_{i, j} G^{A}\left(z_{i}, w_{j}\right)\right) .
\end{aligned}
$$

The theta characteristics $\delta=\left(\delta^{\prime}, \delta^{\prime \prime}\right)$ are given by

$$
\Omega \delta^{\prime}+\delta^{\prime \prime}=\sum_{i} \int_{P}^{z_{i}} \omega_{I}-\sum_{j} \int_{P}^{w_{j}} \omega_{I}-(2 n-1) \Delta+\Omega \alpha^{\prime}+\alpha^{\prime \prime},
$$

where $\left(\alpha^{\prime}, \alpha^{\prime \prime}\right)$ define the spin structure of the $b(d z)^{n}, c(d z)^{1-n}$, and $\Delta$ is the Riemann vector of constants. A more precise chiral version of (5.1) is also in [12], which is of special interest since it shows that the meromorphic propagators $S^{n}\left(z, w ; z_{1}, \ldots, z_{\Upsilon_{n}}\right)$ of $(2.15)$ can all be written in terms of theta functions:

$$
\begin{aligned}
\left\langle\prod_{i=1}^{M+r_{n}} b\left(z_{i}\right) \prod_{j=1}^{M} c\left(w_{j}\right)\right\rangle= & Z_{\Delta}^{-1} \theta[\alpha]\left(\sum_{i} \int_{P}^{z_{i}} \omega_{I}-\sum_{j} \int_{P}^{w_{J}} \omega_{I}-(2 n-1) \Delta\right) \\
& \frac{\prod_{i<j} E\left(z_{i}, z_{j}\right) \prod_{i<j} E\left(w_{i}, w_{j}\right)}{\prod_{i, j} E\left(z_{i}, w_{j}\right)}\left(\frac{\prod \sigma\left(z_{i}\right)}{\prod \sigma\left(w_{j}\right)}\right)^{2 n-1} .
\end{aligned}
$$

Here $\sigma(z)$ is a multi-valued nowhere vanishing holomorphic form of rank $h / 2$. The ratio $\sigma(z) / \sigma(w)$ can be expressed as

$$
\frac{\sigma(z)}{\sigma(w)}=\frac{\theta\left(z-\sum_{1}^{h} \int_{P}^{p_{i}} \omega_{I}-\Delta\right)}{\theta\left(w-\sum_{1}^{h} \int_{P}^{p_{i}} \omega_{I}-\Delta\right)} \prod_{i} \frac{E\left(w, p_{i}\right)}{E\left(z, p_{i}\right)}
$$

for any choice of $h$ points $p_{i}$ on $M$. The factor $Z_{\Delta}^{-1}$ is the partition function of a chiral scalar. The expression (5.3) is anomalous, but the anomalies will cancel in all relevant formulas. The passage from (5.1) to (5.3) requires extraction of the correct conformal and gravitational anomalies from the theta factors and Arakelov Green's functions to produce the holomorphic forms $\sigma\left(z_{i}\right)$ and $\sigma\left(w_{j}\right)$.

As a consequence the propagator of $(2.15)$ is given by (say $n>1$ )

$$
\begin{aligned}
S^{n}\left(z, w ; z_{1}, \ldots, z_{\Upsilon_{n}}\right)= & \frac{1}{E(z, w)} \frac{\theta[\alpha]\left(z-w+\sum_{i} z_{i}-(2 n-1) \Delta\right)}{\theta[\alpha]\left(\sum_{i} z_{i}-(2 n-1) \Delta\right)} \\
& \frac{\prod_{j} E\left(z, z_{j}\right)}{\prod_{j} E\left(w, z_{j}\right)}\left(\frac{\sigma(z)}{\sigma(w)}\right)^{2 n-1} \cdot
\end{aligned}
$$

Explicit expressions for holomorphic $n$-forms can be obtained as well. In fact the 
operator product expansions of the $b, c$ system imply that

$$
\partial_{\bar{w}} S^{n}\left(z, w ; z_{1}, \ldots, z_{Y_{n}}\right)=-2 \pi \delta(z-w)+2 \pi \sum_{1}^{r_{n}} \delta\left(z_{k}-w\right) \phi_{k}^{n}(z)
$$

with $\phi_{k}^{n}(z)$ a basis of holomorphic $n$-forms. Applying (5.5) gives then

$$
\phi_{j}^{n}(z)=\frac{\theta[\alpha]\left(z-z_{j}+\sum_{k} z_{k}-(2 n-1) \Delta\right)}{\theta[\alpha]\left(\sum_{k} z_{k}-(2 n-1) \Delta\right)} \frac{\prod_{k \neq j} E\left(z, z_{k}\right)}{\prod_{k \neq j} E\left(z_{j}, z_{k}\right)}\left(\frac{\sigma(z)}{\sigma\left(z_{j}\right)}\right)^{2 n-1} .
$$

These formulas are useful in some multiloop calculations for string scattering. For our purposes, it is better to proceed inductively as in (2.16), and we shall make use only of non-chiral bosonization as stated in (5.1). For $n>1$ recall that a basis $\phi_{a}^{n+1}$ of holomorphic $(n+1)$ forms can be obtained from a basis $\phi_{a}^{n}$ of holomorphic $n$-forms by

$$
\begin{aligned}
\phi_{a}^{n+1}(z) & =\omega_{z} \phi_{a}^{n}(z), \quad a=1, \ldots, \Upsilon_{n}, \\
\phi_{Y_{n}+b}^{n+1}(z) & =\omega_{z} S^{n}\left(z, R_{b} ; z_{1}, \ldots, z_{Y_{n}}\right)\left(\partial \omega\left(R_{b}\right)\right)^{(n-1) / 2},
\end{aligned}
$$

where the $(2 h-2)$ points $R_{b}$ are the zeroes of the holomorphic form $\omega_{z}$. For $n=1$ the construction (5.8) has to be modified to account for the presence of the $c$ zero mode which is just a constant:

$$
\begin{aligned}
\phi_{I}^{(2)}(z) & =\omega_{z} \omega_{I}(z), \quad I=1, \ldots, h, \\
\phi_{h+a}^{(2)}(z) & =\omega_{z} \partial_{z} \ln \left(\frac{E\left(z, R_{2 h-2}\right)}{E\left(z, R_{a}\right)}\right), \quad a=1, \ldots, 2 h-3 .
\end{aligned}
$$

Here $\omega_{I}$ are a basis of abelian differentials, and $\partial_{z}\left(\ln E\left(z, R_{2 h-2}\right) / E\left(z, R_{a}\right)\right)$ is just the abelian differential of the third kind with poles at $R_{2 h-2}$ and $R_{a}$. Finally for $n=1 / 2$ we consider separately the cases of even and odd spin structures. Generically for even spin structures, the $b, c$ fields of rank $1 / 2$ have no zero mode, and the $(2 h-2)$ holomorphic $3 / 2$ forms can be constructed as in (5.8),

$$
\phi_{a}^{(3 / 2)}(z)=\omega_{z} S^{1 / 2}\left(z, R_{a}\right)\left(\partial \omega\left(R_{a}\right)\right)^{-1 / 4}, \quad a=1, \ldots, 2 h-2
$$

with $S^{1 / 2}(z, w)$ the Szego kernel. On the other hand for generic odd spin structure $\left(\alpha^{\prime}, \alpha^{\prime \prime}\right)$ the $b, c$ fields will have exactly one zero mode $h_{\alpha}$ given by

$$
h_{\alpha}(z)=\left(\sum_{1}^{h} \partial_{I} \theta[\alpha](0, \Omega) \omega_{I}(z)\right)^{1 / 2},
$$

and the $(2 h-2)$ holomorphic $3 / 2$ forms will be obtained by a construction more akin to (5.9),

$$
\begin{aligned}
\phi_{a}^{3 / 2}(z) & =\omega_{z} S^{1 / 2}\left(z, R_{a} ; R, R_{2 h-2}\right)\left(\partial \omega\left(R_{\alpha}\right)\right)^{-1 / 4}, \quad a=1, \ldots, 2 h-3, \\
\phi_{2 h-2}^{3 / 2}(z) & =\omega_{z} h_{\alpha}(z),
\end{aligned}
$$

and $S^{1 / 2}\left(z, w ; R, R^{\prime}\right)$ is the meromorphic $1 / 2$-form in $z$ with poles at $w$ and $R^{\prime}$, and 
a zero at $R$ given by

$$
\begin{aligned}
S^{1 / 2}\left(z, w ; R, R^{\prime}\right) & =\frac{\left\langle b(z) c(w) b(R) c\left(R^{\prime}\right)\right\rangle}{\left\langle b(R) c\left(R^{\prime}\right)\right\rangle} \\
& =\frac{1}{E(z, w)} \frac{\theta[\alpha]\left(z+R-w-R^{\prime}\right)}{\theta[\alpha]\left(R-R^{\prime}\right)} \frac{E(z, R) E\left(w, R^{\prime}\right)}{E(w, R) E\left(z, R^{\prime}\right)}
\end{aligned}
$$

Next, bosonization formulas give determinants of Laplacians in terms of arbitrary insertions at a fixed number of points $z_{i}$ and $w_{j}$. As in the case of zero modes, it is useful to choose these points inductively. We shall make the following choices:

$$
\begin{array}{lll}
\text { tensor rank } & \text { Insertion for } n & \text { Insertion for } n+1 \\
n>1 & \prod_{1}^{r_{n}} b\left(z_{i}\right) & \prod_{1}^{r_{n}} b\left(z_{i}\right) \prod_{1}^{2 h-2} b\left(R_{a}\right) \\
n=1 & \prod_{1}^{h} b\left(z_{i}\right) c(w) & \prod_{1}^{h} b\left(z_{i}\right) \prod_{1}^{2 h-3} b\left(R_{a}\right) \\
n=1 / 2 \text { even spin str. } & \text { No insertion } & \prod_{1}^{2 h-2} b\left(R_{a}\right) \\
n=1 / 2 \text { odd spin str. } & b(R) c\left(R_{2 h-2}\right) & \prod_{1}^{2 h-3} b\left(R_{a}\right) b(R)
\end{array}
$$

With these choices it is readily seen that

$$
\begin{aligned}
\operatorname{det} \phi_{a}^{n+1}\left(z_{b}\right)= & \operatorname{det} \phi_{a}^{n}\left(z_{c}\right) \prod_{1}^{r_{n}} \omega\left(z_{i}\right) \prod_{1}^{2 h-2}\left(\partial \omega\left(R_{a}\right)\right)^{(n+1) / 2} \\
& n>1 \quad \text { or } n=1 / 2, \text { even spin structure } \\
= & \operatorname{det} \phi_{a}^{n}\left(z_{i}\right) \prod_{1}^{h} \omega\left(z_{i}\right) \prod_{1}^{2 h-3} \partial \omega\left(R_{a}\right), \quad n=1 ; \\
= & \omega(R) h_{\alpha}(R)\left(\prod_{1}^{2 h-3} \partial \omega\left(R_{a}\right)\right)^{3 / 4}, \quad n=1 / 2 \text { odd spin. }
\end{aligned}
$$

Finally we need some basic relations between $\omega_{z}$, the Arakelov metric, and the Arakelov Green's function which follow readily from the defining equation $g_{z \bar{z}}^{A}=\left|\omega_{z}\right|^{2} \exp (-2 \sigma(z))$. At general points $z_{j}$ we have

$$
\left|\omega\left(z_{j}\right)\right|^{2}=\exp \left(-\prod_{1}^{2 h-2} G^{A}\left(z_{j}, R_{a}\right)-c\right) g_{z \bar{z}}^{A}
$$

while at the zeroes $R_{a}$ of $\omega_{z}$

$$
\left|\partial \omega\left(R_{a}\right)\right|^{2}=\exp \left(-\sum_{b \neq a} G^{A}\left(R_{b}, R_{a}\right)-c\right)\left(g_{R_{a} \bar{R}_{a}}^{A}\right)^{2} .
$$

Recall that we have chosen the Arakelov metric for convenience, and the constant $c$ as given by (4.3) gives the normalization of $\omega_{z}$.

We can now apply the bosonization formulas (5.1) with the above choices of 
zero modes and insertion points. Since the $R_{a}$ 's are the zeros of an abelian differential, they satisfy $I\left(R_{1}+\cdots+R_{2 h-2}\right)=2 \Delta$, and all theta factors will cancel in ratios $W_{n+1} / W_{n}$. Using (5.15) and (5.16) we can also eliminate explicitly all dependence on the insertion points $z_{j}, w, R$, and just leave dependence on insertion points $R_{a}, a=1, \ldots, 2 h-2$. The net results are

$$
\begin{aligned}
\frac{W_{n+1}}{W_{n}}\left(g_{z \bar{z}}^{A}\right) & =\exp \left(\frac{n}{2} \sum_{a, b=1}^{2 h-2} G^{A}\left(R_{a}, R_{b}\right)\right) \exp (3 n(h-1) c), \quad n \geqq 1, \quad n=1 / 2, \text { even spin } \\
& =\exp \left(\frac{1}{4} \sum_{a, b=1}^{2 h-2} G^{A}\left(R_{a}, R_{b}\right)\right) \frac{\left|h_{\alpha}\left(R_{2 h-2}\right)\right|^{2}}{\left\langle h_{\alpha} \mid h_{\alpha}\right\rangle} \exp (3(h-1) c / 2) \\
n & =1 / 2, \text { odd spin. }
\end{aligned}
$$

These are relations between determinants of different weights for Arakelov metrics. They can be rewritten more explicitly as

$$
\begin{aligned}
\frac{\operatorname{det}^{\prime} \Delta_{n+1}^{-}}{\operatorname{det}^{\prime} \Delta_{n}^{-}}\left(g_{z \bar{z}}^{A}\right)= & \exp \left(-n \sum_{a, b=1}^{2 h-2} G^{A}\left(R_{a}, R_{b}\right)\right) \prod_{1}^{2 n-2}\left|\sqrt{2} g_{R_{a} \bar{R}_{a}}^{A} / \partial \omega\left(R_{a}\right)\right|^{3 n} \\
& \frac{\operatorname{det}\left\langle\phi_{a}^{n+1} \mid \phi_{b}^{n+1}\right\rangle_{\text {Arakelov }}}{\operatorname{det}\left\langle\phi_{a}^{n} \mid \phi_{b}^{n}\right\rangle_{\text {Arakelov }}}, n \geqq 1 \quad \text { or } n=1 / 2 \text {, even spin. }
\end{aligned}
$$

For $n=1 / 2$, odd spin, the relation becomes

$$
\begin{aligned}
\frac{\operatorname{det}^{\prime} \Delta_{3 / 2}^{-}}{\operatorname{det}^{\prime} \Delta_{1 / 2}^{-}}\left(g_{z \bar{z}}^{A}\right)= & \exp \left(-\frac{1}{2} \sum_{a, b=1}^{2 h-2} G^{A}\left(R_{a}, R_{b}\right)\right) \prod_{1}^{2 h-2}\left|\frac{\sqrt{2} g_{R_{a}}^{A} \bar{R}_{a}}{\partial \omega\left(R_{a}\right)}\right|^{3 / 2} \\
& \frac{\left|h_{\alpha}\left(R_{2 h-2}\right)\right|^{2}}{\operatorname{det}\left\langle\phi_{a}^{3 / 2} \mid \phi_{b}^{3 / 2}\right\rangle_{\text {Arakelov }}}, \quad n=1 / 2 .
\end{aligned}
$$

To pass to Mandelstam metrics we note that the defining relation (4.14) and Eqs. (5.15), (5.16) imply

$$
\frac{W_{n+1}}{W_{n}}\left(\left|\omega_{z}\right|^{2}\right)=\frac{W_{n+1}}{W_{n}}\left(g_{z \bar{z}}^{A}\right) \exp \left(-n \sum_{a, b=1}^{2 h-2} G^{A}\left(R_{a}, R_{b}\right)\right)\left(\prod_{1}^{2 h-2} g_{R_{a} \bar{R}_{a}}^{A}\right)^{n} \exp (-4 n(h-1) c) .
$$

Combining (5.17), (5.19) gives the desired result,

$$
\begin{aligned}
& \frac{W_{n+1}}{W_{n}}\left(\left|\omega_{z}\right|^{2}\right)=\left|\prod_{a=1}^{2 h-2} \partial \omega\left(R_{a}\right)\right|^{n}, \quad n \geqq 1 \text { or } n=1 / 2 \text {, even spin structure, } \\
& \frac{W_{3 / 2}}{W_{1 / 2}}\left(\left|\omega_{z}\right|^{2}\right)=\left|\prod_{a=1}^{2 h-2} \partial \omega\left(R_{a}\right)\right|^{1 / 2} \frac{\left|h_{\alpha}\left(R_{2 h-2}\right)\right|^{2}}{\left\langle h_{\alpha} \mid h_{\alpha}\right\rangle_{\text {Arakelov }}}, \quad \text { odd spin structure. }
\end{aligned}
$$

\section{Surfaces with Punctures}

The above analysis can be extended to the case of surfaces with punctures $M^{*}=M-\left\{z_{A}\right\}$, i.e., metrics $\left|\omega_{z}\right|^{2}$, where $\omega_{z}$ has poles at points $z_{A}, A=1, \ldots, N$. As shown by Sonoda, one can still define the ratio $W_{n}\left(\left|\omega_{z}\right|^{2}\right)$ by regularizing the 
Liouville action, the only difference between zeros and poles of $\omega_{z}$ reflecting itself in the relative signs of the Dirac point masses in the curvature. The new subtlety lies rather in the fact that for surfaces with punctures $z_{A}$ the zero modes should also include meromorphic forms with simple poles at $z_{A}$. This difficulty is taken care of by noting that given a coordinate system around each $z_{A}$, a basis of meromorphic forms $m_{A}$ can be singled out by requiring that $m_{A}$ be orthogonal to holomorphic forms and have singularity $\delta_{A B} / z-z_{B}$ near $z_{B}$. The regularized $W_{n}\left(\left|\omega_{z}\right|^{2}\right)$ of (2.11) for a choice of holomorphic zero modes $\phi_{a}^{n}$ can then be viewed as the regularized $W_{n}^{*}\left(\left|\omega_{z}\right|^{2}\right)$ for the basis $\left(\phi_{a}^{n}, m_{A}\right)$ of holomorphic $n$-forms on $M^{*}$. Altogether $W_{n}^{*}\left(\left|\omega_{z}\right|^{2}\right)$ is then a tensor of type $\left(c_{n} / 12-n+1, c_{n} / 12-n+1\right)$ at each $z_{A}$. In particular the ratio $W_{n+1}^{*} / W_{n}^{*}\left(\left|\omega_{z}\right|^{2}\right)$ is a scalar in $z_{A}$. Its dependence on the scale of $\left|\omega_{z}\right|^{2}$ dictate that it be proportional to $\left(\prod_{A} \alpha_{A}\right)^{-2 n}$, where $\alpha_{A}$ is the residue of $\omega_{z}$ at $z_{A}$, which is a scalar. This means that the change of weight formula for Mandelstam metrics with both zeroes and poles should read

$$
\begin{aligned}
\int D(b \bar{b} c \bar{c}) \exp \left(-I_{n+1}\right)\left|\prod_{1}^{M+r_{n}} b\left(z_{i}\right) \prod_{1}^{M} c\left(w_{j}\right)\right|^{2} \\
=\int D(b \bar{b} c \bar{c}) \exp \left(-I_{n+1}\right)\left|\prod_{1}^{M+r_{n}} b\left(z_{i}\right) \prod_{1}^{2 h-2+N} b\left(R_{a}\right) \prod_{1}^{M} c\left(w_{j}\right) \sum_{1}^{N} c\left(z_{A}\right)\right|^{2} \\
\quad \cdot\left|\frac{\prod_{1}^{M} \omega\left(w_{j}\right)}{\prod_{1}^{M+r_{n}} \omega\left(z_{i}\right)}\right|^{2 n} \prod_{1}^{2 n-2+N}\left|\partial \omega\left(R_{a}\right)\right|^{-2 n-1}\left(\prod_{1}^{N} \alpha_{N}\right)^{2 n} .
\end{aligned}
$$

Careful duplication of the arguments for zeroes of $\omega_{z}$ shows that indeed (6.1) holds with precisely this additional factor on the right-hand side. Thus

$$
\begin{aligned}
& \frac{W_{n+1}}{W_{n}}\left(\left|\omega_{z}\right|^{2}\right)=\left|\prod_{a=1}^{2 h-2+N} \partial \omega\left(R_{a}\right)\right|^{n}\left|\prod_{A=1}^{N} \alpha_{A}\right|^{-2 n}, n \geqq 1 n=1 / 2 \text {, even spin structure, } \\
& \frac{W_{3 / 2}}{W_{1 / 2}}\left(\left|\omega_{z}\right|^{2}\right)=\left|\prod_{a=1}^{2 h-2+N} \partial \omega\left(R_{a}\right)\right|^{1 / 2} \frac{\left|h_{\alpha}\left(R_{2 h-2}\right)\right|^{2}}{\left\langle h_{\alpha} \mid h_{\alpha}\right\rangle_{\text {Arakelov }}} \prod_{A=1}^{N}\left|\alpha_{A}\right|^{-1}, \text { odd spin. }
\end{aligned}
$$

Acknowledgements. We would like to thank A. Morozov for asking questions which led to this paper, and the Aspen Center for Physics where part of this work was carried out.

\section{References}

1. D'Hoker, E., Phong, D. H.: Nucl. Phys. B269, 205 (1986); Commun. Math. Phys. 105, 537 (1986); Fried, D.: Invent. Math. 84, 523 (1986); Sarnak, P.: Commun. Math. Phys. 110, 113 (1987); Namazie, A., Rajeev, S.: Nucl. Phys. B277, 332 (1986); Gilbert, G.: Nucl. Phys. B277, 102 (1986); Voros, A.: Commun. Math. Phys. 110, 439 (1987); Gava, E., Iengo, R., Jayaraman, T., Ramachandran, R.: Phys. Lett. 168B, 207 (1986); Wolpert, S.: Commun. Math. Phys. 112, 283 (1987); Gross, D., Periwal, V.: Phys. Rev. Lett. 60, 2105 (1988); Kierlanczyk, K.: M.I.T.Ph.D. thesis, 1986; Baranov, Schwarz, A.: ZETP 42, 340 (1985), Ray, D., Singer, I. M.: Ann. Math. 98, 154 (1973)

2. Knizhnik, V.: Phys. Lett. B180, 247 (1986); Iengo, R., Ivanov, B.: ICTP Preprint IC/87/401 Trieste; 
Morozov, A., Perelomov, A.: Phys. Lett. B197, 115 (1987)

3. Mandelstam, S.: Phys. Rep. C13, 259 (1974). In: Unified string theories. Green, M., Gross, D. (eds.) (1986); In: Second Nobel Symposium on Elementary Particle Physics, 1986.

4. Witten, E.: Nucl. Phys. B268, 253 (1986)

5. Giddings, S., Wolpert, S.: Commun. Math. Phys. 109, 177 (1987)

6. D'Hoker, E., Giddings, S.: Nucl. Phys. B291, 90 (1987)

7. Sonoda, H.: Nucl. Phys. B294, 157 (1987)

8. D'Hoker, E., Phong, D. H.: Rev. Mod. Phys. 60, 917 (1988)

9. Witten, E.: Commun. Math. Phys. 113, 529 (1988)

10. Alvarez-Gaumé, L. et al.: Commun. Math. Phys. 112, 503 (1987)

11. Dugan, M., Sonoda, H.: Nucl. Phys. B289, 227 (1987)

12. Verlinde, E., Verlinde, H.: Nucl. Phys. B288, 357 (1987)

13. Efrat, I.: Commun. Math. Phys. 119, 443 (1988)

14. Arakelov, S.: Math. USSR Izv. 8, 1167 (1974)

Communicated by S.-T. Yau

Received December 15, 1988 
